\title{
On additive involutions and Hamel bases
}

\author{
KAROL BARON
}

\begin{abstract}
We provide an example of a discontinuous involutory additive function $a: \mathbb{R} \rightarrow \mathbb{R}$ such that $a(H) \backslash H \neq \emptyset$ for every Hamel basis $H \subset \mathbb{R}$ and show that, in fact, the set of all such functions is dense in the topological vector space of all additive functions from $\mathbb{R}$ to $\mathbb{R}$ with the Tychonoff topology induced by $\mathbb{R}^{\mathbb{R}}$.
\end{abstract}

Mathematics Subject Classification (2000). Primary 39B52.

Keywords. Additive involution, Hamel basis, linear topological space, Tychonoff topology.

\section{Introduction}

By a Hamel basis of $\mathbb{R}$ we mean (see [2, p. 82]) a basis of the vector space $\mathbb{R}$ over the field $\mathbb{Q}$ of rationals. Inspired by the foot-note on p. 325 of [2] we are interested in discontinuous additive functions $a: \mathbb{R} \rightarrow \mathbb{R}$ which are involutory, i.e., $a \circ a=\operatorname{id}_{\mathbb{R}}$, and such that

$$
a(H) \backslash H \neq \emptyset
$$

for every Hamel basis $H \subset \mathbb{R}$.

\section{Existence}

The following theorem provides an example of a discontinuous involutory additive function $a: \mathbb{R} \rightarrow \mathbb{R}$ such that (1) holds for every set $H \subset \mathbb{R}$ which is linearly independent over $\mathbb{Q}$ and has at least three elements.

Theorem 1. Assume $X$ is a linear space over the field $\mathbb{Q}$ with $\operatorname{dim} X \geq 3$. If $H_{0}$ is a basis of $X, h_{0} \in H_{0}$ and $a: X \rightarrow X$ is the additive function defined by

$$
a\left(h_{0}\right)=h_{0} \quad \text { and } \quad a(h)=-h \quad \text { for } h \in H_{0} \backslash\left\{h_{0}\right\},
$$


then a is involutory,

$$
a(x)+x \in \mathbb{Q} h_{0} \quad \text { for } \quad x \in X,
$$

and (1) holds for every linearly independent set $H \subset X$ with card $H \geq 3$.

Proof. To see that $a(a(x))=x$ for $x \in X$ it is enough to observe that it holds for $x \in H_{0}$.

Let $r: X \rightarrow \mathbb{Q}$ be the function such that

$$
x-r(x) h_{0} \in \operatorname{Lin}\left(H_{0} \backslash\left\{h_{0}\right\}\right) \quad \text { for } x \in X .
$$

Then, according to (2),

$$
a(x)-r(x) h_{0}=a\left(x-r(x) h_{0}\right)=-\left(x-r(x) h_{0}\right)
$$

whence

$$
a(x)=-x+2 r(x) h_{0} \quad \text { for } x \in X .
$$

In particular we have (3),

$$
\text { if } x \in X \text { and } a(x)=x \text {, then } x \in \mathbb{Q} h_{0},
$$

and

$$
\text { if } x \in X \text { and } r(x)=0 \text {, then } a(x)=-x \text {. }
$$

Suppose $H \subset X$ is linearly independent and (1) does not hold, i.e.,

$$
a(H) \subset H .
$$

It follows from (7) and (6) that $r(h) \neq 0$ for $h \in H$ and making use of (4) we see that

$$
h_{0}=\frac{a(h)+h}{2 r(h)} \quad \text { for } h \in H .
$$

We will show that

if $h_{1}, h_{2} \in H \backslash \mathbb{Q} h_{0}$ are different, then $r\left(h_{1}\right)=r\left(h_{2}\right)$ and $h_{1}+h_{2}=2 r\left(h_{1}\right) h_{0}$.

To this end fix arbitrarily different elements $h_{1}, h_{2}$ of $H \backslash \mathbb{Q} h_{0}$. It follows from (8) that

$$
\frac{a\left(h_{1}\right)+h_{1}}{r\left(h_{1}\right)}=\frac{a\left(h_{2}\right)+h_{2}}{r\left(h_{2}\right)}
$$

which jointly with (7) gives

$$
\operatorname{card}\left\{h_{1}, h_{2}, a\left(h_{1}\right), a\left(h_{2}\right)\right\} \leq 3 .
$$

Hence, taking (5) into account and that $a$ is involutory, we have

$$
h_{2}=a\left(h_{1}\right)
$$

whence, by (4) and (10),

$$
h_{2}=-h_{1}+2 r\left(h_{1}\right) h_{0}
$$


and

which ends the proof of (9).

$$
\frac{h_{2}+h_{1}}{r\left(h_{1}\right)}=\frac{a\left(a\left(h_{1}\right)\right)+h_{2}}{r\left(h_{2}\right)}=\frac{h_{1}+h_{2}}{r\left(h_{2}\right)}
$$

Since $H$ is linearly independent, (9) implies that card $H \leq 2$.

Remark 1. Let a be defined as in Theorem 1.

(i) If $h_{1} \in H_{0} \backslash\left\{h_{0}\right\}$, then $h_{0}+h_{1}, h_{0}-h_{1}$ are linearly independent and

$$
a\left(\left\{h_{0}+h_{1}, h_{0}-h_{1}\right\}\right)=\left\{h_{0}+h_{1}, h_{0}-h_{1}\right\} .
$$

(ii) If $X$ is a linear space over a field $\mathbb{K}$ such that $\mathbb{Q}$ is its proper subfield, then

$$
a\left(\alpha h_{0}\right) \neq \alpha a\left(h_{0}\right) \quad \text { for } \alpha \in \mathbb{K} \backslash \mathbb{Q} .
$$

In particular, if $X$ is a linear topological space, then the function

$$
a \mapsto a\left(\alpha h_{0}\right), \quad \alpha \in \mathbb{R},
$$

is discontinuous.

Proof. Part (i) is obvious, (11) follows from (3) and (2), and the continuity of (12) implies $a\left(\alpha h_{0}\right)=\alpha a\left(h_{0}\right)$ for $\alpha \in \mathbb{R}$.

\section{Density}

Fix a linear topological space $X, X \neq\{0\}$, and consider the space $X^{X}$ of all functions from $X$ into $X$ with the usual addition and multiplication by scalars and with the Tychonoff topology. Clearly $X^{X}$ is a linear topological space. Put

$$
\mathcal{A}=\left\{a \in X^{X}: a \text { is additive }\right\}
$$

and consider $\mathcal{A}$ with the topology induced by $X^{X}$.

Theorem 2. The sets

$\left\{a \in \mathcal{A}: a \circ a=\mathrm{id}_{X}, a\right.$ is discontinuous and (1) holds for every uncountable set $H \subset X$ which is linearly independent over $\mathbb{Q}\}$,

$\left\{a \in \mathcal{A}: a \circ a=\operatorname{id}_{X}, a\right.$ is discontinuous and $a(H)=H$ for a basis $H$ of the vector space $X$ over the field $\mathbb{Q}\}$

are dense in $\mathcal{A}$.

For the proof the following lemma from [1] will be used.

Lemma 1. A subset $\mathcal{D}$ of $\mathcal{A}$ is dense in $\mathcal{A}$ if and only if for any $M \in \mathbb{N}$, for any $h_{1}, \ldots, h_{M}, h^{\prime}{ }_{1}, \ldots, h^{\prime}{ }_{M} \in X$ being linearly independent over $\mathbb{Q}$, and for any neighbourhood $U \subset X$ of zero there exists $a \in \mathcal{D}$ such that

$$
a\left(h_{m}\right) \in U+h_{m}^{\prime} \quad \text { for } m \in\{1, \ldots, M\} .
$$


In fact we will apply the following corollary resulting from this lemma.

Corollary 1. If $\mathcal{D} \subset \mathcal{A}$ and for any $M \in \mathbb{N}$ and for any $h_{1}, \ldots, h_{M}, h^{\prime}{ }_{1}, \ldots, h^{\prime}{ }_{M}$ $\in X$ being linearly independent over $\mathbb{Q}$ there exists $a \in \mathcal{D}$ such that

$$
a\left(h_{m}\right)=h_{m}^{\prime} \quad \text { for } m \in\{1, \ldots, M\},
$$

then $\mathcal{D}$ is dense in $\mathcal{A}$.

Proof of the density of the set (13). Fix $M \in \mathbb{N}$ and $h_{1}^{o}, \ldots, h_{M}^{o}, h_{1}^{o^{\prime}}, \ldots, h_{M}^{o^{\prime}} \in$ $X$ linearly independent over $\mathbb{Q}$. Let $H_{0}$ be a basis of the vector space $X$ over the field $\mathbb{Q}$ such that $h_{1}^{o}, \ldots, h_{M}^{o}, h_{1}^{o^{\prime}}, \ldots, h_{M}^{o^{\prime}} \in H_{0}$ and define the additive function $a: X \rightarrow X$ by putting

$$
a\left(h_{m}^{o}\right)=h_{m}^{o^{\prime}}, a\left(h_{m}^{o^{\prime}}\right)=h_{m}^{o} \quad \text { for } m \in\{1, \ldots, M\},
$$

and

$$
a(h)=-h \quad \text { for } h \in H_{0} \backslash\left\{h_{1}^{o}, \ldots, h_{M}^{o}, h_{1}^{o^{\prime}}, \ldots, h_{M}^{o^{\prime}}\right\} .
$$

Clearly, $a \circ a=\operatorname{id}_{X}$.

Let $r_{1}, \ldots, r_{M}, r^{\prime}{ }_{1}, \ldots, r^{\prime}{ }_{M}: X \rightarrow \mathbb{Q}$ be the functions such that

$x-\left(\sum_{m=1}^{M} r_{m}(x) h_{m}^{o}+\sum_{m=1}^{M} r_{m}^{\prime}(x) h_{0}^{\prime}\right) \in \operatorname{Lin}_{\mathbb{Q}}\left(H_{0} \backslash\left\{h_{1}^{o}, \ldots, h_{M}^{o}, h_{1}^{o^{\prime}}, \ldots, h_{M}^{o^{\prime}}\right\}\right)$

for $x \in X$. Then

$$
a(x)=-x+\sum_{m=1}^{M}\left(r_{m}(x)+r_{m}^{\prime}(x)\right)\left(h_{m}^{o}+h_{m}^{o^{\prime}}\right) \quad \text { for } x \in X .
$$

In particular,

$$
a(x)+x \in \operatorname{Lin}_{\mathbb{Q}}\left\{h_{1}^{o}, \ldots, h_{M}^{o}, h_{1}^{o^{\prime}}, \ldots, h_{M}^{o^{\prime}}\right\} \quad \text { for } x \in X
$$

and so $a$ is discontinuous.

Suppose $H \subset X$ is uncountable, linearly independent over $\mathbb{Q}$, and (1) does not hold. Take different $h_{1}, \ldots, h_{2 M+1} \in H$ such that

$$
h_{k}+h_{l} \notin \operatorname{Lin}_{\mathbb{Q}}\left\{h_{1}^{o}, \ldots, h_{M}^{o}, h_{1}^{o^{\prime}}, \ldots, h_{M}^{o^{\prime}}\right\} \quad \text { for } k, l \in\{1, \ldots, 2 M+1\} .
$$

It follows from (15) that

$$
a\left(h_{1}\right)+h_{1}, \ldots, a\left(h_{2 M+1}\right)+h_{2 M+1}
$$

are linearly dependent over $\mathbb{Q}$ and so are

$$
h_{1}, \ldots, h_{2 M+1}, a\left(h_{1}\right), \ldots, a\left(h_{2 M+1}\right) .
$$

Hence, taking (7) into account and that $a$ is injective, we have

$$
a\left(h_{k}\right)=h_{l}
$$

for some $k, l \in\{1, \ldots, 2 M+1\}$. This and (15) give

$$
h_{k}+h_{l}=h_{k}+a\left(h_{k}\right) \in \operatorname{Lin}_{\mathbb{Q}}\left\{h_{1}^{o}, \ldots, h_{M}^{o}, h_{1}^{o^{\prime}}, \ldots, h_{M}^{o^{\prime}}\right\}
$$


which contradicts (16). The contradiction obtained shows that $a$ belongs to the set (13) and applying Corollary 1 we conclude the proof.

Proof of the density of the set (14). Fix $M \in \mathbb{N}$ and $h_{1}, \ldots, h_{M}, h^{\prime}{ }_{1}, \ldots, h^{\prime}{ }_{M} \in$ $X$ linearly independent over $\mathbb{Q}$. Let $H$ be a basis of the vector space $X$ over the field $\mathbb{Q}$ such that $h_{1}, \ldots, h_{M}, h^{\prime}{ }_{1}, \ldots, h^{\prime}{ }_{M} \in H$ and define the additive function $a: X \rightarrow X$ by putting

$$
a\left(h_{m}\right)=h_{m}^{\prime}, a\left(h_{m}^{\prime}\right)=h_{m} \quad \text { for } m \in\{1, \ldots, M\}
$$

and

$$
a(h)=h \quad \text { for } h \in H \backslash\left\{h_{1}, \ldots, h_{M}, h_{1}^{\prime}, \ldots, h_{M}^{\prime}\right\} .
$$

Then $a \circ a=\mathrm{id}_{X}$,

$$
a(x)-x \in \operatorname{Lin}_{\mathbb{Q}}\left\{h_{1}, \ldots, h_{M}, h_{1}^{\prime}, \ldots, h_{M}^{\prime}\right\} \quad \text { for } x \in X
$$

and $a(H)=H$. Hence $a$ is in the set (14) and applying Corollary 1 we find that this set is dense in $\mathcal{A}$.

\section{Acknowledgement}

The research was supported by the Silesian University Mathematics Department (Iterative Functional Equations and Real Analysis program).

Open Access. This article is distributed under the terms of the Creative Commons Attribution License which permits any use, distribution, and reproduction in any medium, provided the original author(s) and the source are credited.

\section{References}

[1] Baron, K., Volkmann, P.: Dense sets of additive functions, Seminar LV, No. 16. http:// www.math.us.edu.pl/smdk (2003)

[2] Kuczma, M.: An introduction to the theory of functional equations and inequalities. Cauchy's equation and Jensen's inequality. In: Gilányi, A. (ed.) 2nd edn. Birkhäuser Verlag, Basel (2009)

\section{Karol Baron}

Instytut Matematyki

Uniwersytet Ślạski

ul. Bankowa 14

40-007 Katowice

Poland

e-mail: baron@us.edu.pl

Received: November 7, 2012

Revised: December 6, 2012 